\title{
Analysis of eutrophication state and trend for lakes in China
}

\author{
JIN XIANGCAN \\ Chinese Research Academy of Environmental Sciences, Beijing, China
}

\begin{abstract}
ABSRACT
The article analyzes the present state and trend of eutrophication of lakes in China and concludes that lakes throughout the country are commonly undergoing the process of eutrophication: most of urban lakes are facing hypertrophication, many mediumsized lakes are of eutrophic state, some lakes even approaching to hypertrophic level. The five large freshwater lakes are in the condition of eutrophication, especially Lake Caohu and Lake Taihu are already in the state of eutrophication, water quality is deteriorating and ecosystem is destroyed. According to domestic and foreign experiences of the successful demonstrations in eutrophication and pollution treatment, this article puts forward the theory of combining source control with ecological restoration, which as the guidance for eutrophication control of lakes in China.
\end{abstract}

Key words: lake, eutrophication, countermeasure

\section{GENERAL SITUATION OF LAKES IN CHINA AND ITS MAIN ENVIRONMENTAL PROBLEMS}

China is a country boasting of many lakes, with over 24,880 lakes, 2300 of them with a surface higher than 1 $\mathrm{km}^{2}$. The total area of all the lakes reaches $70,988 \mathrm{~km}^{2}$, accounting for about $0.8 \%$ of the total area of the country, and most of lakes distributes in the East Plain, Qingzang Altiplano, Mengxin Altiplano, Yungui Altiplano, and Northeast Plain-hills, also called Five Big Lakes Zone. All lakes are with a total storage capacity of 707.7 billion $\mathrm{m}^{3}$, of which freshwater storage capacity amounts to 225 billion $\mathrm{m}^{3}$. Besides, there are 86,825 reservoirs, with a total storage capacity of 413 billion $\mathrm{m}^{3}$. The two add to a total freshwater capacity of $6380 \times$ $10^{8} \mathrm{~m}^{3}$, forming one of the most important freshwater resources in the country, playing a major role in supplying drinking water for the people as well as water for industrial and agricultural production, and aquaculture, travel industry, improving climate, generating electricity etc.

Owing to various natural geography and climate condition of Chinese lakes, in the recent several decade years discharge of large quantities of pollutants and human activities of irrational exploitation, environmental problems of lakes shows complexity and diversity. There are five main environmental problems: eutrophication, pollution of organic pollutants, salting of lakes in the west of China, shrinking of area and reduction of water quantity of lakes and destruction of ecosystem. But at present eutrophication is the most important environmental problem in many lakes and thus brings a tremendous influence on sustainable development of society and economy in lake regions.

\section{PRESENT STATE OF TREND EUTROPHICATION OF LAKES}

Investigation of 1980s and 1990s shows eutrophic lakes was mainly located in the middle and lower stream of Changjiang River, Yungui Altiplano, part of Northeast Plain-hills and Mengxin Altiplano. But all the urban lakes almost have been in the eutrophic level because the urban lakes are small and seriously destroyed by the domestic wastewater.

Owing to the fact that people have not realized the frailty of the lake ecosystem and lack of environmental awareness, human activities such as land reclaimation and destruction of plants around lakes, discharge of large quantities of industrial and agricultural waste water into lakes, and irrational development and utilization of natural resources of lakes, and so on have greatly affected the environment of lakes, which now face many environmental problems. And lakes throughout the country are commonly undergoing the process of eutrophication. As a result, the cycling of the ecosystem of many lakes is damaged, causing great losses to production and people's life in lake regions. Figure 1 shows trophic state of over 50 Chinese lakes. Eutrophic and hypertrophic lakes account for $66 \%$ and hypertrophic lakes amounts to $22 \%$. Therefore, lake eutrophication has become an important environmental problem now.

Investigation shows that many freshwater lakes, even some lakes in the district where people seldom lived have accounted to the eutrophic state and most of them have been in the high trophic level. The characteristics of trophic state of lakes follow as:

1) The five largest freshwater lakes have been in the eutrophic level, they have been generally facing eutrophication with high nutrients (Tabs 1a, 1b), for 


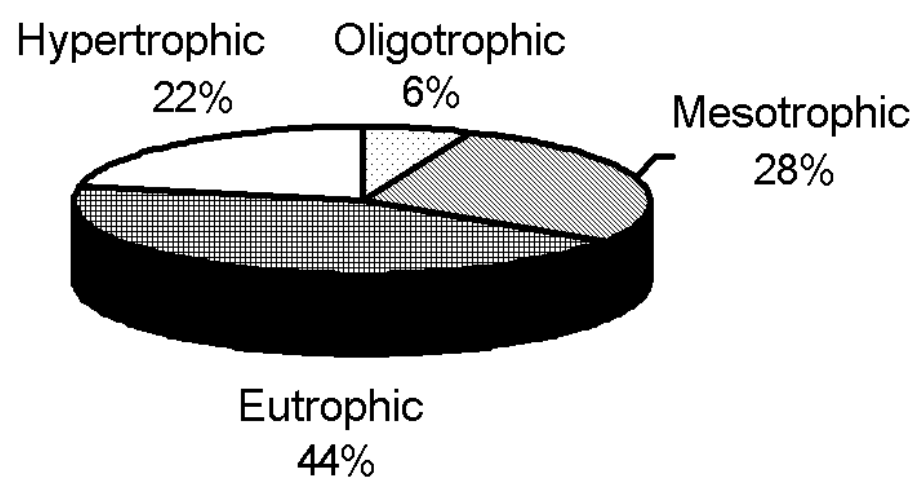

Fig. 1. Trophic state of main lakes in China.

Tab. 1a. Trophic state and estimation of over 50 Lakes in China. **Biggest freshwater lakes.

\begin{tabular}{|c|c|c|c|c|c|c|c|c|c|c|c|c|c|}
\hline & \multicolumn{10}{|c|}{ Index $\left(\mathrm{C}_{\mathrm{i}}\right)$} & \multirow[t]{2}{*}{$\mathrm{TSI}_{\mathrm{G}}$} & \multicolumn{2}{|c|}{ Trophic conditions } \\
\hline & $\begin{array}{l}\text { Chl- } a \\
\mu \mathrm{g} \mathrm{l} 1^{-1}\end{array}$ & $\begin{array}{c}\mathrm{TP} \\
\mathrm{mg} \mathrm{l}^{-1}\end{array}$ & $\begin{array}{c}\mathrm{TN} \\
\mathrm{mg} \mathrm{l}^{-1}\end{array}$ & SD & $\begin{array}{l}\mathrm{COD}_{\mathrm{Mn}} \\
\mathrm{mg} \mathrm{l}^{-1}\end{array}$ & $\begin{array}{l}\mathrm{BOD}_{5} \\
\mathrm{mg} \mathrm{l}^{-1}\end{array}$ & $\begin{array}{l}\mathrm{DO} \\
\mathrm{mg} \mathrm{l}^{-1}\end{array}$ & Py & $\begin{array}{l}\mathrm{NH}_{3}-\mathrm{N} \\
\mathrm{mg} \mathrm{l}^{-1}\end{array}$ & $\mathrm{pH}$ & & Estimated & Practical \\
\hline \multicolumn{14}{|l|}{ East Plain Lakes Zone } \\
\hline **Poyang L. & 1.34 & 0.094 & 0.67 & 0.65 & 2.05 & 2.39 & 8.43 & 47.60 & & & 39.31 & Meso-eutrophic & Meso-trophic \\
\hline **Dongting L. & 1.68 & 0.119 & 1.168 & 0.39 & 2.13 & 0.78 & 9.71 & & & & 39.14 & Meso-eutrophic & Meso-trophic \\
\hline **Taihu L. & 5.35 & 0.052 & 1.11 & 0.70 & 3.16 & 1.69 & 9.00 & & & & 41.57 & Meso-eutrophic & Meso-eutrophic \\
\hline **Hongze L. & & 0.130 & 1.39 & 0.38 & 2.85 & & 7.11 & & & & 46.00 & Meso-eutrophic & Meso-eutrophic \\
\hline **Caohu L. & 15.01 & 0.088 & 1.43 & 0.25 & 3.76 & 2.33 & & & & & 46.06 & Eutrophic & Eutrophic \\
\hline Qiandao L. & 3.72 & 0.057 & 0.40 & 5.83 & 1.85 & 0.81 & & & & & 35.6 & Meso-trophic & Meso-trophic \\
\hline Gucheng L. & 4.05 & 0.055 & 1.73 & 0.34 & 2.65 & 1.03 & 7.27 & & & & 42.93 & Meso-eutrophic & Meso-eutrophic \\
\hline Nansi L. & 3.73 & 0.21 & 3.38 & 0.50 & 21.38 & & 6.79 & & & & 51.40 & Eutrophic & Europhic \\
\hline Dianshan L. & 5.98 & 0.088 & 1.95 & 0.57 & 4.30 & 1.73 & 8.90 & 50 & & & 43.26 & Meso-eutrophic & Meso-eutrophic \\
\hline Baiyangdian L. (80s) & & 0.082 & 2.30 & & 5.00 & 4.24 & 11.68 & & 0.396 & & 47.79 & Eutrophic & Eutrophic \\
\hline \multicolumn{14}{|l|}{$\begin{array}{l}\text { Yangui Altiplano Lake Zone } \\
\text { Dianchi Lake }\end{array}$} \\
\hline Caohai L. & 77.41 & 0.504 & & 0.40 & 14.74 & & & & & & 61.84 & Hypertrophic & Hypertrophic \\
\hline Inner L. & 101.74 & 41.401 & & 0.28 & 16.58 & & & & & & 67.00 & Hypertrophic & Hypertrophic \\
\hline Average of Dianchi L. & 49.70 & 0.270 & & 0.60 & 8.40 & & & & & & 56.43 & Hypertrophic & Hypertrophic \\
\hline $\begin{array}{l}\text { Average (except for } \\
\text { InnerL. and Caohai L.) }\end{array}$ & 16.76 & 0.123 & & 0.65 & 7.07 & & & & & & 50.26 & Eutrophic & Eutrophic \\
\hline Erhai L.(1995) & & 0.03 & 0.29 & 3.00 & & 0.57 & & 127 & & & 34.52 & Meso-trophic & Meso-trophic \\
\hline Cibi L. in Dali (1995) & & 0.02 & 0.29 & 1.40 & & 0.53 & & 135 & & & 35.07 & Meso-trophic & Meso-trophic \\
\hline Xihu L. in Dali (1995) & & 0.02 & 0.46 & 1.00 & & 1.51 & & 148 & & & 38.88 & Meso-eutrophic & Meso-trophic \\
\hline Haixihai L. in Dali (1995) & & 0.01 & 0.56 & 1.20 & & 0.90 & & 218 & & & 37.31 & Meso-trophic & Meso-trophic \\
\hline Tianchi L. in Dali (1992) & & 0.02 & 0.51 & 2.30 & & 1.55 & & 133 & & & 37.30 & Meso-trophic & Meso-trophic \\
\hline Jianhu L. in Dali (1993) & & 0.03 & 0.33 & 1.20 & & 1.37 & & 72 & & & 37.56 & Meso-trophic & Meso-trophic \\
\hline Mutunhai L. in Dali (1994) & & 0.02 & 0.28 & 0.8 & & 0.86 & & 254 & & & 37.90 & Meso-trophic & Meso-trophic \\
\hline Dayindian L. in Dali (1995) & & 0.02 & 0.49 & 1.70 & & 1.50 & & 611 & & & 39.54 & Meso-eutrophic & Meso-eutrophic \\
\hline Xinyun L. (1990) & & 0.045 & 0.67 & 1.50 & 3.98 & 2.16 & & 418.3 & & & 41.96 & Meso-eutrophic & Meso-eutrophic \\
\hline Yilong L. (1988) & & 0.122 & & 0.62 & 22.18 & 7.18 & 5.22 & & 1.23 & 8.93 & 55.39 & Hypertrophic & Hypertrophic \\
\hline The east of Yilong L. & & 0.08 & & 0.70 & 18.92 & 9.24 & 6.25 & & 1.06 & 8.91 & 54.09 & Hypertrophic & Hypertrophic \\
\hline The middle of Yilong L. & & 0.154 & & 0.65 & 20.51 & 6.59 & 6.18 & & 1.34 & 8.95 & 55.32 & Hypertrophic & Hypertrophic \\
\hline The west of Yilong L. & & 0.133 & & 0.50 & 27.31 & 11.15 & 3.24 & & 1.30 & 8.92 & 58.54 & Hypertrophic & Hypertrophic \\
\hline Qilu L. & & 0.09 & 2.187 & 0.40 & 8.29 & 3.55 & 7.30 & & 0.59 & 8.98 & 52.09 & Hypertrophic & Hypertrophic \\
\hline Fuxian L.1988) & & 0.0098 & 0.15 & 7.10 & 0.80 & 0.57 & & 18.02 & & & 25.98 & Oligo-trophic & Oligo-trophic \\
\hline Qionghai L. (1988) & 0.649 & 0.137 & 1.21 & 2.13 & 1.488 & 0.50 & 6.70 & 14.44 & 0.003 & & 32.12 & Meso-trophic & Meso-trophic \\
\hline
\end{tabular}

example, concentration of $\mathrm{TN}$ is above 10 times higher. Now Lake Taihu and Lake Caohu are already in the eutrophic state, with some waters even approaching to hypereutrophic level. And the other three lakes may also enter into eutrophication in the condition of higher nutrient load.

2) The urban lakes are facing serious eutrophication. According the investigation of many years, the urban lakes have been facing serious eutrophication with extremely high concentration of TN, TP, Chl- $a$ and low transparency (SD) in water bodies and mainly in the hypertrophic level accompanying the serious organic pollution with very high concentration of $\mathrm{COD}_{\mathrm{Mn}}$ and $\mathrm{BOD}_{5}$ in waters, exceeding the Grade V water(GB3838-88) (Tab. 1).

3) Most of medium-sized lakes are already of the eutrophic state. Lots of medium-sized lakes are already of the eutrophic state with TN and TP approaching or 
Tab. 1b. Trophic state and estimation of over 50 Lakes in China.

\begin{tabular}{|c|c|c|c|c|c|c|c|c|c|c|c|c|c|}
\hline & \multicolumn{10}{|c|}{ Index $\left(\mathrm{C}_{\mathrm{i}}\right)$} & \multirow[t]{2}{*}{$\mathrm{TSI}_{\mathrm{G}}$} & \multicolumn{2}{|c|}{ Trophic conditions } \\
\hline & $\begin{array}{l}\text { Chl- } a \\
\mu \mathrm{g} 1^{-1}\end{array}$ & $\begin{array}{c}\mathrm{TP} \\
\mathrm{mg} \mathrm{l}^{-1}\end{array}$ & $\begin{array}{c}\mathrm{TN} \\
\mathrm{mg} \mathrm{1}^{-1}\end{array}$ & SD & $\begin{array}{l}\mathrm{COD}_{\mathrm{Mn}} \\
\mathrm{mg} \mathrm{l}^{-1}\end{array}$ & $\begin{array}{l}{ }_{\mathrm{n}} \mathrm{BOD}_{5} \\
\mathrm{mg} \mathrm{l}^{-1}{ }_{1}\end{array}$ & $\begin{array}{l}\mathrm{DO}^{5} \\
\mathrm{mg} \mathrm{l}^{-1}\end{array}$ & Py & $\begin{array}{l}\mathrm{NH}_{3}-\mathrm{N} \\
\mathrm{mg} \mathrm{l}^{-1}\end{array}$ & $\mathrm{pH}$ & & Estimated & Practical \\
\hline $\begin{array}{l}\text { Quingzang Lake Zone } \\
\text { Qinghai L. } \\
\text { Namucuo L. }\end{array}$ & 0.042 & $\begin{array}{c}0.02 \\
0.031\end{array}$ & $\begin{array}{c}0.08 \\
0.411\end{array}$ & $\begin{array}{l}8.00 \\
9.00\end{array}$ & 1.41 & & 5.63 & & & & $\begin{array}{l}29.27 \\
27.70\end{array}$ & $\begin{array}{l}\text { Oligo-Meso-trophic } \\
\text { Oligo-Meso-trophic }\end{array}$ & $\begin{array}{l}\text { Meso-trophic } \\
\text { Oligo-trophic }\end{array}$ \\
\hline $\begin{array}{l}\text { Mengxin Altiplano } \\
\text { Boshiteng L. } \\
\text { Caiwobao L. } \\
\text { Wulungu L. } \\
\text { Hulun L. } \\
\text { Wuliangsuhai L. } \\
\text { Hasuhai L. } \\
\text { Dihai L. (1989) }\end{array}$ & $\begin{array}{c}\text { Zone } \\
5.22 \\
0.099 \\
0.031 \\
\\
4.45 \\
15.51 \\
32.56\end{array}$ & $\begin{array}{c}0.018 \\
5.93 \\
1.98 \\
0.140 \\
0.067 \\
0.090 \\
0.22\end{array}$ & $\begin{array}{l}0.92 \\
0.27 \\
3.30 \\
1.88 \\
1.873 \\
1.21 \\
2.25\end{array}$ & $\begin{array}{c}1.74 \\
5.21 \\
13.36 \\
0.47 \\
1.21 \\
0.88 \\
0.61\end{array}$ & $\begin{array}{c}6.02 \\
1.58 \\
1.41 \\
13.36 \\
6.44 \\
7.68 \\
132.47\end{array}$ & $\begin{array}{l}1.20 \\
7.76 \\
8.86 \\
1.41 \\
1.86 \\
2.31 \\
2.18\end{array}$ & $\begin{array}{c}7.30 \\
22.00 \\
8.10 \\
9.19 \\
6.43 \\
7.35 \\
7.18\end{array}$ & $\begin{array}{c}36.3 \\
1.32 \\
0.55 \\
230 \\
1218 \\
1387 \\
111.75\end{array}$ & 0.025 & & $\begin{array}{l}38.00 \\
51.05 \\
43.89 \\
46.41 \\
44.70 \\
46.89 \\
51.55\end{array}$ & $\begin{array}{c}\text { Meso-trophic } \\
\text { Eutrophic } \\
\text { Meso-eutrophic } \\
\text { Eutropjhic } \\
\text { Meso-eutrophic } \\
\text { Eutrophic } \\
\text { Eutrophic }\end{array}$ & $\begin{array}{c}\text { Meso-trophic } \\
\text { Eutrophic } \\
\text { Meso-eutrophic } \\
\text { Eutrophic } \\
\text { Meso-eutrophic } \\
\text { Eutrophic } \\
\text { Eutropic }\end{array}$ \\
\hline $\begin{array}{l}\text { North-East plain hills La } \\
\text { Songhua L. } \\
\text { Tianchi L. (in Jilin) } \\
\text { Xingkai L. } \\
\text { Jingpo L. } \\
\text { Wudalianchi L. (90) }\end{array}$ & ke Zone & $\begin{array}{l}0.10 \\
0.40 \\
0.419\end{array}$ & $\begin{array}{l}0.55 \\
0.98 \\
1.67\end{array}$ & $\begin{array}{l}0.60 \\
1.32 \\
1.15\end{array}$ & $\begin{array}{l}5.28 \\
0.78 \\
5.54 \\
7.00 \\
7.97\end{array}$ & $\begin{array}{l}1.20 \\
1.96 \\
1.21\end{array}$ & $\begin{array}{l}8.90 \\
8.50 \\
8.89 \\
7.97\end{array}$ & $\begin{array}{c}292 \\
3.11 \\
225.15\end{array}$ & $\begin{array}{l}0.064 \\
0.170\end{array}$ & 7.7 & $\begin{array}{l}41.87 \\
23.82 \\
40.43 \\
46.25 \\
47.92\end{array}$ & $\begin{array}{l}\text { Meso-eutrophic } \\
\text { Oligo-trophic } \\
\text { Meso-eutrophic } \\
\text { Eutrophic } \\
\text { Eutophic }\end{array}$ & $\begin{array}{c}\text { Meso-eutrophic } \\
\text { Oligo-trophic } \\
\text { Meso-eutrophic } \\
\text { Eutophic } \\
\text { Eutophic }\end{array}$ \\
\hline $\begin{array}{l}\text { Urban Lakes } \\
\text { Luhu L. } \\
\text { Liuhua L. } \\
\text { Dongshan L. } \\
\text { Liwan L. } \\
\text { Xihu L. (in Hangzhou) } \\
\text { Cihu L. } \\
\text { DongHu L. (in Wuhan) } \\
\text { Moshui L. (in Wuhan) } \\
\text { Xuanwu L. (in Nanjing) } \\
\text { Gantang L. (in Jiujiang) } \\
\text { Nanhu L. (in Changchun) } \\
\text { Mogu L. (1988) } \\
\text { Dianchi L. (Caohai) }\end{array}$ & $\begin{array}{c}86.40 \\
239.53 \\
132.00 \\
149.65 \\
64.80 \\
11.20 \\
15.5 \\
156.59 \\
103.00 \\
37.60 \\
113.11 \\
32.12 \\
77.41\end{array}$ & $\begin{array}{c}0.22 \\
0.530 \\
0.420 \\
0.620 \\
0.17 \\
0.090 \\
0.125 \\
0.740 \\
0.478 \\
0.240 \\
0.529 \\
0.21 \\
0.504\end{array}$ & $\begin{array}{l}3.04 \\
6.38 \\
6.15 \\
8.53 \\
3.06 \\
2.20 \\
2.50 \\
16.05 \\
3.50 \\
1.73 \\
5.45 \\
2.33 \\
\end{array}$ & $\begin{array}{l}0.38 \\
0.20 \\
0.29 \\
0.31 \\
0.53 \\
0.67 \\
0.80 \\
0.15 \\
0.25 \\
0.56 \\
0.19 \\
0.62 \\
0.40\end{array}$ & $\begin{array}{c}9.68 \\
23.36 \\
11.74 \\
14.43 \\
7.18 \\
3.94 \\
12.56 \\
13.6 \\
8.95 \\
26.17 \\
78.28 \\
\\
\\
14.74\end{array}$ & $\begin{array}{c}8.71 \\
16.51 \\
14.26 \\
17.50 \\
4.84 \\
3.68 \\
3.50 \\
25.71 \\
10.70 \\
6.41 \\
9.76\end{array}$ & $\begin{array}{c}9.66 \\
11.44 \\
6.93 \\
4.74 \\
10.15 \\
7.20 \\
\\
4.26 \\
8.64 \\
8.03 \\
8.58 \\
9.88\end{array}$ & $\begin{array}{l}1000 \\
4767 \\
1193\end{array}$ & 0.76 & & $\begin{array}{l}55.75 \\
63.80 \\
60.71 \\
44.70 \\
54.43 \\
46.87 \\
51.52 \\
62.87 \\
58.53 \\
54.00 \\
63.30 \\
52.09 \\
61.84\end{array}$ & $\begin{array}{l}\text { Hypertrophic } \\
\text { Hypertrophic } \\
\text { Hypertrophic } \\
\text { Hypertrophic } \\
\text { Hypertrophic } \\
\text { Eutrophic } \\
\text { Eutrophic } \\
\text { Hypertrophic } \\
\text { Hypertrophic } \\
\text { Hypertrophic } \\
\text { Hypertrophic } \\
\text { Eutrophic } \\
\text { Hypertrophic }\end{array}$ & $\begin{array}{l}\text { hypertrophic } \\
\text { hypertrophic } \\
\text { hypertrophic } \\
\text { hypertrophic } \\
\text { hypertrophic } \\
\text { Eutophic } \\
\text { Eutophic } \\
\text { hypertrophic } \\
\text { hypertrophic } \\
\text { hypertrophic } \\
\text { hypertrophic } \\
\text { Eutophic } \\
\text { hypertrophic }\end{array}$ \\
\hline $\begin{array}{l}\text { Reservoirs } \\
\text { Miyun R. (1990) } \\
\text { Yuqiao R. } \\
\text { Dahuofang R. (88-91) } \\
\text { Gaozhou R. }\end{array}$ & $\begin{array}{c}3.02 \\
6.56 \\
5.433 \\
0.739\end{array}$ & $\begin{array}{c}0.0175 \\
0.02 \\
0.06 \\
0.022\end{array}$ & $\begin{array}{l}50.115 \\
1.24 \\
1.09 \\
0.369\end{array}$ & $\begin{array}{l}2.15 \\
1.97 \\
2.10 \\
2.11\end{array}$ & $\begin{array}{l}2.41 \\
3.36 \\
4.20 \\
1.64\end{array}$ & $\begin{array}{l}1.68 \\
3.02\end{array}$ & $\begin{array}{c}7.98 \\
10.26 \\
7.402\end{array}$ & $\begin{array}{l}58.18 \\
99500 \\
267.65\end{array}$ & $\begin{array}{l}0.063 \\
0.153\end{array}$ & & $\begin{array}{l}33.02 \\
46.49 \\
41.37 \\
32.60\end{array}$ & $\begin{array}{l}\text { Meso-trophic } \\
\text { Eutrophic } \\
\text { Meso-eutrophic } \\
\text { Mesotrophic }\end{array}$ & $\begin{array}{l}\text { Meso-trophic } \\
\text { Eutrophic } \\
\text { Meso-eutrophic } \\
\text { Mesotrophic }\end{array}$ \\
\hline
\end{tabular}

exceeding the eutrophication transition concentration and some waters even reaching hypertrophic level (Lake Dianchi and Lake Erhai).

The concentration of $\mathrm{COD}_{\mathrm{Mn}}, \mathrm{BOD}_{5}, \mathrm{TP}$ and $\mathrm{TN}$ is high in eutrophic lakes. Secchi disk transparency is lower than $1.0 \mathrm{~m}$, and in most of them even lower than $0.5 \mathrm{~m}$. Water quality is more and more deteriorating and the lake ecosystems appear to be greatly damaged.

Also the problem of nutrient elements released from sediment is serious. For example, $29 \%$ area of Lake Taihu is coverd with $0.1 \mathrm{~m}$ or more of highly contaminated sediment. The total storage capacity amounts to 69.1 million $\mathrm{m}^{3}$. Algal nutrients (especially phosphorus) are the most important elements released from sediments, together with the refloating of small organic granule. There are so much nutrient elements in sediments that Lake Tajihu can still maintain an high level of eutrophication, even if all the external loading is reduced to zero.

As a consequence of the extremely high trophic condition, ecosystem in many lakes is commonly dam- aged due to deterioration of water quality, lowering of transparency, and destruction of lakeshore vegetation and wetland. The change of biological community accompanying the raising of pollutants and nutrients level is hereafter briefly summarized.

Phytoplankton densities largely increase, diversity of phytoplankton community declines, and dominant species of phytoplankton community change into species characteristic of eutrophic environments as cyanobacteria, which dominate in almost all the lake.

In eutrophic lakes phytoplankton produce high blooms and seasonal changes are reduced. In the hypertrophic and eutrophic lakes average yearly densities of phytoplanktonic algae vary between 1,000,000 and $10,000,000$ ind $1^{-1}$ and in the urban lakes the average algae per year amounts to between 10,000,000 and 100,000,000 ind $\mathrm{l}^{-1}$ (Tab. 2).

Diversity of phytoplankton community decrease. In eutrophic lakes seasonal change of composition of phytoplankton community tends to simplicity and the general tendency is that the higher is the concentration of nutrients in lakes simpler is the species composition 
Tab. 2. Yearly average concentration of Chl-a, number of individuals and biomass of phytoplankton for some lakes in China.

\begin{tabular}{|c|c|c|c|c|}
\hline & $\begin{array}{l}\text { Chl- } a \\
\left(\mu \mathrm{g} \mathrm{l}^{-1}\right)\end{array}$ & $\begin{array}{l}\text { n. ind. } \\
\left(\times 10^{4} 1^{-1}\right)\end{array}$ & $\begin{array}{l}\text { Biomass } \\
\left(\mathrm{mg} \mathrm{l}^{-1}\right)\end{array}$ & Trophic state \\
\hline Luhu L. (Guangzhou) & 86.40 & 9695.7 & & Hypertrophic \\
\hline Liuhua L. (Guangzhou) & 239.53 & 1561.0 & 51.94 & Hypertrophic \\
\hline Dongshan L. (Guangzhou) & 132.00 & 4505.1 & & Hypertrophic \\
\hline s Liwan L. (Guangzhou) & 150.00 & 5664.0 & & Hypertrophic \\
\hline Xihu L. (Hangzhou) & 64.80 & 4319.8 & & Hypertrophic \\
\hline Moshui L. (Wuhan) & 157.00 & 9692.7 & & Hypertrophic \\
\hline ฮี Donghu L. (Wuhan) & 15.50 & 322.4 & & Eutrophic \\
\hline O Xuanwu L. (Nanjing) & 103.00 & 4767.0 & & Hypertrophic \\
\hline Gantang L. (Jiujiang) & 37.60 & 5316.3 & & Hypertrophic \\
\hline Nanhu L. (Changchun) & 113.11 & 1237.4 & & Hypertrophic \\
\hline Caohai in Dianchi L. & 139.00 & 6467.0 & & Hypertrophic \\
\hline Outer of Dianchi L. & 23.80 & 1364.9 & & Eutrophic \\
\hline Poyang L. (Jiangxi) & 1.34 & 65.45 & & Meso-trophic \\
\hline Taihu L. (Jiangsu) & 5.35 & 32.1 & 5.86 & Meso-eutrophic \\
\hline Caohu L. (Anhui ) & 15.01 & 214.1 & & Eutrophic \\
\hline Erhai L. (Yunnan) & & 562.3 & 4.66 & Meso-trophic \\
\hline Qilu L. (Yunnan) & & 290.9 & & Hypertrophic \\
\hline Bositeng L. (Xinjiang) & 5.22 & 340.9 & 1.36 & Mesotrophic \\
\hline Wulungu L. (Xinjiang) & 0.031 & 630.3 & 1.34 & Meso-eutrophic \\
\hline Xika L. (Heilongiiang) & & & 3.67 & Meso-eutrophic \\
\hline Jingpo L.(Heilongjang) & 9.62 & 376.1 & 9.80 & Eutrophic \\
\hline Wudalianchi L. (Heilongjiang) & & 211.7 & 6.17 & Eutrophic \\
\hline Gucheng L. (Jiangshu) & 4.05 & 162.9 & & Meso-eutrophic \\
\hline Nansi L. (Shandong) & 3.83 & 232.4 & & Eutrophic \\
\hline Dianshan L. (Shanghai) & 7.05 & 55.2 & & Meso-eutrophic \\
\hline Fulun L. (Neimeng) & & 3430.5 & 8.10 & Eutrophic \\
\hline Wuliangsuhai L. (Neimeng) & 4.45 & 1218.0 & 2.69 & Meso-eutrophic \\
\hline Daihai L. (Neimeng) & 32.56 & 1127.3 & 1.19 & Meso-eutrophic \\
\hline Qionghai (Sichuan) & 0.70 & 12.4 & & Mesotrophic \\
\hline
\end{tabular}

Tab. 3. Seasonal succession of dominant species of phytoplankton in some of eutrophic lakes in China. Legend: Ana = Anabaena; Ank = Ankistrodesmus; Aph = Aphanocapsa $;$ Apha = Aphanizomenon; Ast = Asterionella $;$ Bin $=$ Binuclearia $;$ Chla $=$ Chlamydomonas; Chlo $=$ Chlorella $;$ Cho $=$ Chodatella $;$ Chrc $=$ Chrcoococcus $;$ Chrm $=$ Chromulina $;$ Chro $=$ Chroomonas $;$ Clo $=$ Closterium; $\mathrm{Coe}=$ Coelastrum; $\mathrm{Cos}=$ Cosmarium; $\mathrm{Cru}=$ Crucigenia $;$ Cry $=$ Cryptomonas; Cyc $=$ Cyclotella; Dac $=$ Dactylococcopsis $;$ Din = Dinobryon $;$ Epi = Epithemia $;$ Eug = Euglena $;$ Fra = Fragilaria $;$ Lyn = Lyngbya $;$ Mel = Melosira $;$ Mer $=$ Merismopedia $;$ Mic $=$ Microcystis $;$ Nav = Navicula $;$ Ooc $=$ Oocystis $;$ Osc $=$ Oscillatoria $;$ Ped $=$ Pediastrum $;$ Pho $=$ Phormidium $;$ Pla Planktosphaeria $;$ Rap = Raphidiopsis; Sce = Scenedesmus; Sch = Schroederia $;$ Syn $=$ Synedra $;$ Tra $=$ Trachelomonas $;$ Eud = Eudorina $;$ Anop $=$ Anabaenopsis $; \mathrm{Chl}=$ Chloroccum $;$ Nos $=$ Nostoc .

\begin{tabular}{|c|c|c|c|c|c|}
\hline Lake & Spring & Summer & Autumn & Winter & Trophic state \\
\hline Cihu L. & Nav,Frg,Cyc,Sce,Ped & Sch,Chro,Osc,Mer,Dac & Osc,Mer,Ooc,Ped,Chla & Cry,Nav & Eutrophic \\
\hline Gantang L. & Mer,Dac & Mer,Dac & Mer,Dac & Mer,Dac & Hypertrophic \\
\hline Moshui L. & Sce,Mer,Cyc & Mic,Sce,Mer & Mic,Cys & Cyc,Mer,Chlo & Hypertrophic \\
\hline Xihu L. & Osc,Syn,Apha & Lym,Sce & Lyn,Ose & Osc & Eutrophic \\
\hline Nanhu L. & Mic,Mer & Mic & Mic & & Hypertrophic \\
\hline Luhu L. & Osc, Chro & Osc,Dac & Rap,Osc & Rap,Osc & Hypertrophic \\
\hline Liuhua L. & Mic & Mic & Mic,Osc & Mic,Osc & Hypertrophic \\
\hline Liwan L. & Cyc,Sce & Sce, Cyc & Sce,Eug & Sce,Cyc,Cry & Hypertrophic \\
\hline Dongshan L. & Cyc,Cry & $\mathrm{Cyc}$ & Cyc,Chro & Cos,Osc & Hypertrophic \\
\hline Honghu L. & Chlo,Eug,Ank,Eud & Chlo,Chro,Eug,Chl & Cos,Osc & Chl & Eutrophic \\
\hline Caohu L. & Chro,Mic,Cry & Mic,Ana & Mic,Chro & Cyc,Mic,Chro & Eutrophic \\
\hline Hulun L. & & Mic,Ana,Chrc,Apha,Mer,Coe & Mic & Mic & Eutrophic \\
\hline Wuliangsuhai L. & Chla,Sce,Mer,Chlo & Mer,Chrc,Mic,Coe,Ana & Mic,Sym,Ana,Lym,Coe,Sce & Sce,Mic & Eutrophic \\
\hline Dianchi L. & Osc,Mic,Cyc,Syn & Osc,Chrc,Cyc,Cry,Sce,Eug & Cyc,Cry,Chla,Mica,Apha & Osc,Mic,Apha,Mica,Eug & Eutrophic \\
\hline Mogu L. & Osc & Osc,Dac & Osc & Osc & Hypertrophic \\
\hline
\end{tabular}

of phytoplankton community and lower bio-diversity. In some lakes such as Gantang Lake composition of phytoplankton community is very simple and only one of algae species takes absolute advantage all over the year. In some lakes such as Luhu Lake, Liuhua Lake and Xuanwu Lake etc. only one of algae take advantage in most seasons (Tab. 4).
Dominant species change to the species indicating the eutrophication. Table 4 shows that in eutrophic lakes the dominant species are species characteristic of the eutrophic waters. In many lakes the quantities of the dominant species increase sharply, which leads to "waters bloom" (Tab. 3). 
Tab. 4. Composition and succession of aquatic plants community in Caohai of Lake Dianchi. $\mathrm{Y}=$ yes; numerical value in the bracket means percent which this community accounts for all the area of all the aquatic plants.

\begin{tabular}{lccccc}
\hline Community type & $1950 \mathrm{~s}$ & $1960 \mathrm{~s}$ & $1970 \mathrm{~s}$ & $1980 \mathrm{~s}$ & $1990 \mathrm{~s}$ \\
\hline Emerged macrophytes community & & & & & \\
$\quad$ Phragmites communis & $\mathrm{Y}(1.1)$ & $\mathrm{Y}(1.2)$ & $\mathrm{Y}(0.2)$ & $\mathrm{Y}$ & $\mathrm{Y}$ \\
Zizania caduciflora & $\mathrm{Y}(1.1)$ & $\mathrm{Y}(1.1)$ & $\mathrm{Y}(0.3)$ & $\mathrm{Y}$ & $\mathrm{Y}$ \\
Shoenoplectus tabernaemontani & $\mathrm{Y}(1.7)$ & $\mathrm{Y}(1.2)$ & $\mathrm{Y}(0.1)$ & & \\
Acorus calamus & $\mathrm{Y}(1.1)$ & $\mathrm{Y}(0.1)$ & $\mathrm{Y}(0.01)$ & & \\
$\begin{array}{l}\text { Echinochloa crusgallia } \\
\text { Hydrophyta natantia community }\end{array}$ & $\mathrm{Y}(1.1)$ & $\mathrm{Y}(0.6)$ & $\mathrm{Y}(0.01)$ & & \\
Lemna minor & $\mathrm{Y}(1.1)$ & $\mathrm{Y}(0.4)$ & $\mathrm{Y}(0.004)$ & $\mathrm{Y}(0.001)$ & \\
A. imbricata & & & $\mathrm{Y}(0.2)$ & $\mathrm{Y}(0.001)$ & $\mathrm{Y}(0.01)$ \\
Eichhornia crassipes & & $\mathrm{Y}(1.2)$ & $\mathrm{Y}(18.6)$ & $\mathrm{Y}(57.6)$ \\
Nymphaeoides peltatum & $\mathrm{Y}(4.3)$ & 1 & $\mathrm{Y}(0.02)$ & & \\
Submerged macrophytes community & & & & & \\
Ottelia acuminata & $\mathrm{Y}(40.4)$ & $\mathrm{Y}(46.9)$ & & & \\
Vallisneria spiralis & $\mathrm{Y}(14.2)$ & $\mathrm{Y}(19.4)$ & $\mathrm{Y}(6.9)$ & & \\
Chara vulgaris & $\mathrm{Y}(17.1)$ & $\mathrm{Y}(16.2)$ & $\mathrm{Y}(0.02)$ & & \\
Ceratophyllum demersum & $\mathrm{Y}(2.8)$ & $\mathrm{Y}(2.5)$ & $\mathrm{Y}(1)$ & \\
Myriophyllum spicatum & $\mathrm{Y}(9.1)$ & $\mathrm{Y}(14.1)$ & $\mathrm{Y}(2.1)$ & $\mathrm{Y}(1.4)$ & $\mathrm{Y}(14.1)$ \\
Potamogeton malainus & & $\mathrm{Y}(7.1)$ & $\mathrm{Y}(3.4)$ & & \\
Potamogeton mackianus & $\mathrm{Y}(3.4)$ & $\mathrm{Y}(4.8)$ & $\mathrm{Y}(3.5)$ & & \\
Potamogeton pectinatus & & $\mathrm{Y}(10.4)$ & $\mathrm{Y}(10.1)$ & $\mathrm{Y}(15.3)$ \\
Potamogeton cripus & $\mathrm{Y}(1.1)$ & $\mathrm{Y}(2.5)$ & $\mathrm{Y}(1.39)$ & $\mathrm{Y}(0.01)$ & $\mathrm{Y}(4)$ \\
Total communities & $\mathrm{Y}(14)$ & $\mathrm{Y}(15)$ & $\mathrm{Y}(16)$ & $\mathrm{Y}(6)$ & $\mathrm{Y}(4)$ \\
\hline
\end{tabular}

Tab. 5. Main composition and change of submerged macrophytes community in various trophic state in Dianchi and Erhai lakes.

\begin{tabular}{|c|c|c|c|}
\hline & n. species & Dominant species & Trophic state \\
\hline \multicolumn{4}{|c|}{ Dianchi Lake } \\
\hline $1950 \mathrm{~s}$ & 44 & O. acuminata, $C$. vulgaris, $V$. spiralis, $M$. spicatum, $P$. maackianus, $D$. demersum, $P$. cripus & Oligotrophic \\
\hline $1960 \mathrm{~s}$ & & $\begin{array}{l}\text { O. acuminata, V. spiralis, C. vulgaris, } M \text {. spicatum, P. malainus, } P \text {. maackianus, D. demersum, } \\
P . \text { cripus }\end{array}$ & Oligotrophic \\
\hline $1970 \mathrm{~s}$ & 30 & P. pectinatus, $V$. spiralis, $P$. maackianus, $P$. malainus, $M$. spicatum, $P$.cripus, $C$. demersum & Mesotrophic \\
\hline $1980 \mathrm{~s}$ & 20 & P. pectinatus, $M$. spicatum, $P$. cripus & Eutrophic \\
\hline $1990 \mathrm{~s}$ & 12 & P. pectinatus, M. spicatum & Hypertrophic \\
\hline \multicolumn{4}{|c|}{ Erhai Lake } \\
\hline $1950 \mathrm{~s}$ & & P. pectinatus, $N$. marina, O. acuminata & Oligotrophic \\
\hline $1960 \mathrm{~s}$ & & & Oligotrophic \\
\hline $1970 \mathrm{~s}$ & 18 & H. verticillata, C. demersum, $P$. maackianus, $P$. lucens & Oligo-mesotrophic \\
\hline $1980 \mathrm{~s}$ & 15 & $H$. verticillata, $V$. spiralis, $P$. maackianus, $C$. demersum & Mesotrophic \\
\hline $1990 \mathrm{~s}$ & 13 & P. maackianus, $V$. spiralis, $Z$. palustris, $H$. verticillata, $C$. demersum & Mesotrophic \\
\hline
\end{tabular}

Accompanying the evolution toward hypertrophic status of lakes, the area of macrophyte gradually shrink. For example, before 1970s Lake Dianchi showed a good water quality with $100 \%$ coverage of aquatic vascular bundle plants, and in outer of Lake Dianchi with SD of over $2 \mathrm{~m}$ and $90 \%$ coverage of various aquatic vascular bundle plants. But after the middle of 1970 s, due to gradual intensity of human activities, Lake Dianchi changes to eutrophication and water quality rapidly deteriorated, leading some species to die out, community composition became more simple. In 1990s the area covered by aquatic plants accounted for only $1.8 \%$ of the total lake area.

In 1950s Lake Dianchi have multiple community types: Chara vulgaris community, Ottelia acuminata community, Vallisneria spiralis community, Myriophyllum spicatum community, Potamogeton malainus community, Acorus calamus community, Scirpus validus community, E. crusgallia community, Phrag- mites communis community, and Potamogeton crispus + M. spicatum + Hydrilla verticillata $+C$. demersum community etc. After 1970s Ottelia acuminata community died out, in succession $C$. vulgaris community died out, owing to inning and repairing of lake shore $A$. calamus and $S$. tabernaemontani community deracinated too, $V$. spiralis community, $P$. malainus community and $P$. cripus $+M$. spicatum community were died gradually, $P$. pectinatus community with high tolerance for pollution evolved, and E. crassipes community took advantage. In 1990s in Caohai of Lake Dianchi $P$. pectinatus community of submerged macrophytes distributed in one or two sites; As for hydrophyta natantia, $E$. crassipes community took advantage with big biomass, and Alternanthera sessilis, Oenanthe japonica, A. imbricate and $L$. minor etc. distributed sporadically in E. crassipes community (Tab. 5).

During the oligotrophic state, there were 44 species of submerged macrophytes in Lake Dianchi and 18 spe- 
Tab. 6. Trophic state and estimation of 34 lakes in China (1978-1980).

\begin{tabular}{lccc}
\hline & Oligotrophic & Mesotrophic & Eutrophic \\
\hline Number & 4 & 16 & 14 \\
$\%$ & 11.76 & 47.06 & 41.2 \\
Area $\left(\mathrm{km}^{2}\right)$ & 3354.6 & 95929 & 5220.6 \\
$\%$ & 3.2 & 91.8 & 5.0 \\
\hline
\end{tabular}

Tab. 7. Trophic state and estimation of over 50 lakes in China (1987-1989).

\begin{tabular}{lccc}
\hline & Oligotrophic & Mesotrophic & Eutrophic \\
\hline Number & 1 & 7 & 14 \\
$\%$ & 4.5 & 31.8 & 63.6 \\
Area $\left(\mathrm{km}^{2}\right)$ & 29.5 & 2493 & 3084.9 \\
$\%$ & 0.53 & 44.45 & 55.0 \\
\hline
\end{tabular}

Tab. 8. Pollutant load $\left(\mathrm{t}^{-1}\right)$ of some lakes in China.

\begin{tabular}{lcccccccc}
\hline & \multicolumn{2}{c}{ Present } & pollutant discharge & & & \multicolumn{3}{c}{ Maximum allowable load } \\
\cline { 2 - 3 } \cline { 7 - 8 } & TP & TN & COD $_{\mathrm{Cr}}$ & & TP & TN & COD $_{\mathrm{Cr}}$ \\
\hline Dianchi L. & 1021 & 8981 & 41,672 & & 356 & 5012 & 5754 \\
Taihu L. & 5168 & 72,017 & 224,032 & & 587 & 2167 & 59,532 \\
Caohu L. & 2677 & 26,802 & 66,773 & & 225 & 5400 & 36,036 \\
Erhai L. & 122 & 1154 & & & & & \\
Yuqiao L. & 228 & 4458 & & & 28 & 888 & \\
\hline
\end{tabular}

cies in Lake Erhai. In the mesotrophic state the number of species declined to 30 species in Lake Dianchi and 13 species in Lake Erhai. Such species as O. acuminata and $C$. vulgaris disappeared. In the hypertrophic state the number of species of Lake Dianchi declined to only 12 , sporadically distributed in a few areas of the lake shore and with small scale (Tab. 6).

Some species with strong tolerance for pollution rapidly increased in density and biomass, while other species gradually declined and, in general, the distribution area of aquatic plants becoming small. For example, $P$. pectinatus come into being only one dominant species community with absolutely preponderant biomass, distribution area and high frequency, leading to $P$. pectinatus covering all the lake.

Frequent emergency of "water blooms" in many urban lakes of China may do serious harm to the lake. First it may produce obstacle to water function of lakes, then it will have impacts on aquaculture and scenic tourism, and algae toxin will endanger the health of people in the lake basin. In early 1980s microcystin (one of the algae toxin) was separated from Lake Donghu in Wuhan. The microcystin was also detected in the drinking water on Tongan (Fujian province) where has high rates of liver cancer. The highest concentration of microcystin in Lake Taihu was $38.5{\mu \mathrm{g}^{-1}}^{-1} 2001$.

The trend of the eutrophic lakes in China is quite rapid. Table 7 shows trophic state of 34 lakes in China. Most of lakes were of in middle- trophic state, account for $91.8 \%$. Eutrophic lakes account for 5.0\% (Tab. 6). In only ten years, oligo-trophic lakes change to be middletrophic lakes. The percent decrease from 3.2 to 0.53 .
Middle-trophic lakes change to be eutrophic lakes, the percent increase from 5.0 to 55.01 (Tab. 7). In 1996, Eutrophic and hypertrophic lakes account for $85 \%$. Therefore, lake eutrophication in China has become an important environmental problems at present.

Excessive discharge of pollutants into lake is one of the important causes of eutrophication of lakes in China. Table 8 shows that now the ratio between present pollutant discharge and the maximal allowable load of the lake are of 3 to 10 in many lakes, and the value is even higher in urban lakes, which lead to deterioration of water quality and eutropohication of lake waters gradually.

\section{COUNTERMEASURE FOR LAKE EUTROPHICATION CONTROL}

All kinds of irrational activities and excessive nutrient load into the lake are the main causes of lake eutrophication and ecological disorder. Therefore, we should stop all kinds of irrational activities first, then take effective measures to restore lake ecosystem gradually.

Taking into consideration the domestic and foreign experience, the strategy to control eutrophication is hereafter described.

1) To combine source control with ecological restoration

Pollution sources are obviously the most direct reason of lake eutrophication, so control pollution sources is regarded as the preliminarily step. But lake is a lived waterbody and we could not control eutrophication ef- 
fectively with the single measure of source control. Lake eutrophication treatment requires to take not only source control measures but also ecological restoration measures. Ecological restoration mainly pay attention to rehabilitation of aquatic plant in the shallow waters, restoration of lakeshore in the water-land crisscross area, and replantation of terrestrial ecological zone in erosion area. Only by ecological restoration measures, lake ecosystem could be restored to normal cycle and eutrophication could be controlled basically.

2) To protect lake from the point of view of the whole catchment

Lake is only part of a ecosystem of the whole lake catchment. Therefore to maintain normal ecosystem requires to control and protect lake from the point of view of the whole catchment.

\section{3) The synthetical treatment and management}

Synthetical treatment and managementis proved to be more rapid and effective measure in prevention and treatment of lakes.

\section{CONSULTED LITERATURE}

Jin Xiangcan et al. 1995. Lakes in China - Research of their environment (I). China Ocean Press.

Jin Xiangcan et al. 1990. Eutrophication of lakes in China. China Environmental Science Press.

Nanjing Institute of Geography and Lake, CAS. 1989. Survey of Chinese Lakes. Science Press.

Wuqinglong, Wangyunfei. 1999. Analysis on succession of bio-community in Lake Erhai. Lakes Sciences, 3: 267-273.

Yuguoying et al. 2000. Relation between succession of aquatic plants and change of water environment. Lakes Sciences, 12(1): 73-79.

Sea and Lakes Society in Prefecture Jiangsu. 1987. Sea and Lake Science Paper Collection. Agriculture Press, Beijing, China.

Jin Xiangcan, Tuqingying et al. 1990. Investigation of the Environment of Lakes and Reservoirs in China. Chinese Environmental Science Press, Beijing, China.

Morikiro Aizaki \& Harukuni Tachibana. 1990. Limnlogical Comparison of Characteristics of Water Quality in Chinese and Japanese Lakes, Proceedings of Symposium on "Limnological Comparison of Chinese and Japanese Eutrophic lakes" at Hokkaido University: 95-96. 